\title{
LA MESURE DE FLUX INTEGRES DE NEUTRONS THERMIQUES AU MOYEN DE COMPTEURS DE GEIGER-MÜLLER A CATHODES ACTIVABLES
}

\author{
par Maurice DARRES et Charles PICCO * \\ (manuscrit reçu le is janvier ig68)
}

\begin{abstract}
RÉSUMÉ
Dans le cas des neutrons thermiques, on abandonne la géométrıe cyınarıque pour adopter des cathodes formées par deux disques parallèles: la perturbation du flux est plus faible, les caractéristiques électriques restant de bonne qualité. Des cathodes de cuivre, de dysprosium et d'or ont été expérimentées dans les neutrons thermiques du réacteur "Siloette", et d'une source de $7 \mathrm{Ci}$ de Pu$\alpha$-Be, les fluences étant déterminées par la méthode d'activation de feuilles d'or (à nu et sous cadmium). Pour les durées d'irradiation inférieures à 0,3 fois la période du radionucléide produit, on peut mesurer des flux intégrés compris entre $4.10^{5} \mathrm{n} \mathrm{cm}^{-2}$ (dysprosium) et $17.10^{10} \mathrm{n} \mathrm{cm}^{-2}$ (cuivre) ce qui correspond à des doses absorbées dans le tissu biologique mou comprises entre 0,25 $\mathrm{m}$ rad et 100 rads.
\end{abstract}

Dans la mesure par activation des flux de neutrons, on irradie l'élément activable, et on le porte ensuite près d'un système de comptage. La géométrie de comptage doit être parfaitement définie et reproductible. On a donc à résoudre un certain nombre de problèmes liés à l'angle solide et à la rétrodiffusion, entre autres.

Il est intéressant de grouper le matériau activable et le détecteur, d'où l'intérêt de compteurs à cathodes activables, en particulier en radioprotection, où il est souhaitable d'effectuer une lecture très rapide des doses absorbées.

Des chercheurs de notre laboratoire ont montré que des compteurs de Geiger à cathodes cylindriques activables, de conception classique, permettent de mesurer des doses intégrées de neutrons rapides [2] [9]. Nous étudions ici l'adaptation de tels détecteurs au cas de flux de neutrons thermiques.

* Centre de Physique Atomique et Nucléaire de la Faculté des Sciences de Toulouse, Ir 8 route de Narbonne, 3 I-Toulouse. 


\section{SÉLECTION DU MATÉRIAU ACTIVABLE (tableau I)}

Le matériau constituant la cathode doit répondre à un certain nombre de conditions impératives :

- posséder une section efficace élevée pour les neutrons thermiques,

- donner un radionucléide de période assez longue pour permettre d'effectuer la mesure et que l'activité soit sensiblement proportionnelle au flux intégré et assez courte pour ne pas augmenter le bruit de fond lors des mesures ultérieures,

- ne pas comporter d'impuretés ou de constituants activables de très longues périodes, qui rendraient irrécupérable le détecteur, tables.

- permettre la réalisation d'une électrode de qualités mécaniques accep-

Nous avons sélectionné le cuivre, le dysprosium et l'or. Le tableau I résume leurs caractéristiques nucléaires.

TABLEAU 1

CARACTÉristiques D'ACTIVATION DES ÉLÉMENTS SÉLECTIONNÉS. D'Après [3]

\begin{tabular}{|c|c|c|c|c|c|}
\hline Elément & $\begin{array}{l}\text { Isotope } \\
\text { (abondance } \\
\text { en } \% \text { en } \\
\text { masse) }\end{array}$ & $\begin{array}{c}\sigma \\
\text { activation } \\
\text { (barns) } \\
\text { pour neutrons } \\
\text { thermiques }\end{array}$ & Réactions & $\begin{array}{c}\text { Période } \\
\text { du } \\
\text { produit }\end{array}$ & $\begin{array}{c}\text { Particules } \\
\text { émises } \\
(\mathrm{MeV}) \\
(*)\end{array}$ \\
\hline Cuivre & $\begin{array}{l}{ }_{23}^{63} \mathrm{Cu}(69, \mathrm{r}) \\
{ }_{29}^{65} \mathrm{Cu}(30,9)\end{array}$ & $\begin{array}{l}4,3 \\
I, 8\end{array}$ & $\begin{array}{l}(n, \gamma) \\
(n, \gamma)\end{array}$ & $\begin{array}{l}\mathrm{r} 2,87 \mathrm{~h} \\
5,15 \mathrm{mn}\end{array}$ & $\begin{array}{l}\beta^{-}: 0,57 \\
\beta^{+}: 0,66 \\
\gamma: 1,34 \\
\beta^{-}: 2,69-1,59 \\
\gamma: 1,14\end{array}$ \\
\hline Dysprosium & ${ }_{66}^{164}$ Dy $(28,18)$ & $\begin{array}{r}2000 \\
800\end{array}$ & $\begin{array}{l}(n, \gamma){ }^{165 m} \mathrm{Dy} \\
(\mathrm{n}, \gamma){ }^{165} \mathrm{Dy}\end{array}$ & $\begin{array}{l}\mathrm{r}, 3 \mathrm{mn} \\
140 \mathrm{mn}\end{array}$ & $\begin{array}{l}\beta^{-}: 0,87 \\
\gamma: 0,108 \text { à } 0,56 \\
\beta^{-}: 1,19 \text { à } 1,28 \\
\gamma: 0,095 \text { à } 0,711\end{array}$ \\
\hline Or & ${ }_{79}^{197} \mathrm{Au}(100)$ & $9^{8,8}$ & $(\mathrm{n}, \gamma)$ & $2,7 \mathrm{j}$ & $\begin{array}{l}\beta^{-}: 0,9^{6}-0,28 \\
\gamma: 0,4^{12-0,69}\end{array}$ \\
\hline
\end{tabular}

(*) Pour les rayons $\beta$, on donne l'énergie maximale du spectre.

\section{FORME DONNÉE AU DÉTECTEUR}

Ce point est d'une très grande importance : l'introduction du détecteur perturbe le flux des neutrons thermiques, par les effets bien connus de dépression de la densité des neutrons, et d'autoblindage [8] [10] [I I ] [13]. Si l'effet est important, l'activation de la cathode varie d'un point à un autre; les mesures sont difficiles à interpréter.

Cette perturbation est d'autant plus forte que le détecteur est plus grand. 
Les compteurs cylindriques ne conviennent pas. Des cathodes constituées par deux disques parallèles assurent une forme plus ramassée (fig. I).

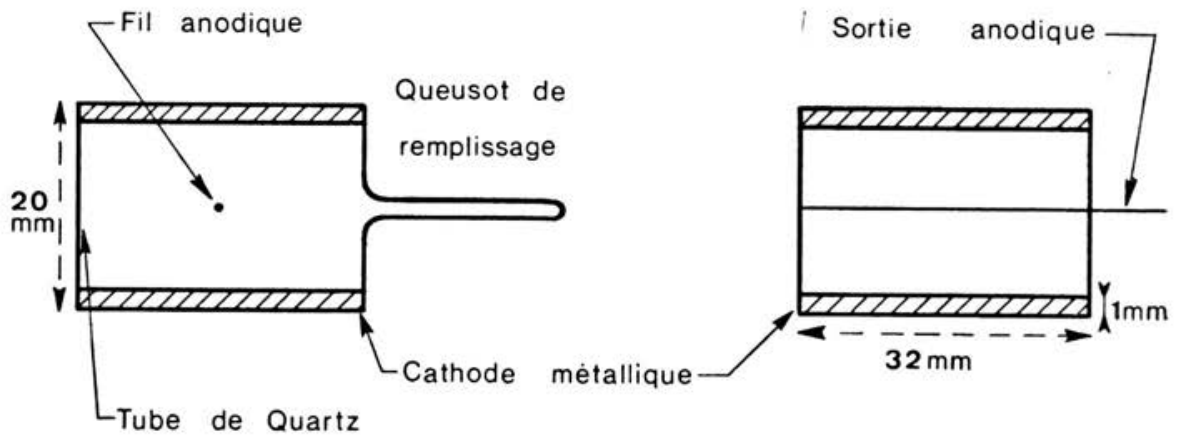

FIG. 1. - Détecteur à cathodes planes et parallèles.

Ces disques (épaisseur : I $\mathrm{mm}$, diamètre : $3,2 \mathrm{~cm}$ ) sont séparés par un tube de quartz long de $2 \mathrm{~cm}$; le quartz ne donne pas, par activation, de radionucléides de période longue. C'est pourquoi nous l'avons préféré au verre. L'anode est un fil de tungstène de $0, \mathrm{I} \mathrm{mm}$ de diamètre, parallèle aux disques et placé à $\mathrm{I} \mathrm{cm}$ de chacun. L'étanchéité est assurée par de l'araldite, produit qui est insensible aux neutrons thermiques. Aucune activation parasite des éléments de structure (quartz, araldite, remplissage gazeux) n'a été constatée au cours des expériences.

\section{CARACTÉRISTIQUES DE COMPTAGE (tableau 2)}

Le tableau 2 correspondant à une surtension $\mathrm{V}-\mathrm{V}_{\mathrm{G}}$ de 100 volts, montre que les compteurs, sans être de qualité comparable à celle des détecteurs cylin-

TABLEAU 2 - CARACTÉRISTIQUES DE COMPTAGE DES DÉTECTEURS EMPLOYÉs.

\begin{tabular}{|c|c|c|c|}
\hline Nature de la cathode & Cuivre & $\begin{array}{l}\text { Dyspro- } \\
\text { sium }\end{array}$ & Or \\
\hline Seuil de Geiger VG (volts) $\ldots \ldots \ldots \ldots \ldots \ldots \ldots \ldots$ & I 320 & I 260 & I 300 \\
\hline Tension de fonctionnement $\mathrm{V}$ (volts) $\ldots \ldots \ldots \ldots \ldots \ldots$ & I 420 & 1360 & I 400 \\
\hline Surtension V-VG (volts) $\ldots \ldots \ldots \ldots \ldots \ldots \ldots \ldots \ldots$ & 100 & 100 & 100 \\
\hline Pente du palier (pour cent par roo volts) $\ldots \ldots \ldots \ldots \ldots$ & 10 & 12 & I I \\
\hline Hauteur de l'impulsion pour $\mathrm{V}$ (volts) $\ldots \ldots \ldots \ldots \ldots \ldots$ & 0,6 & 0,6 & 0,7 \\
\hline Longueur du palier (volts) $\ldots \ldots \ldots \ldots \ldots \ldots \ldots \ldots$ & 320 & 320 & 260 \\
\hline Bruit de fond $\left(\mathrm{mn}^{-1}\right)$ pour $\mathrm{V} \ldots \ldots \ldots \ldots \ldots \ldots \ldots$ & 25 & 40 & 40 \\
\hline Temps mort (microsecondes) pour $\mathrm{V} \ldots \ldots \ldots \ldots \ldots \ldots$ & 60 & 60 & 60 \\
\hline
\end{tabular}


driques [4], possèdent des caractéristiques honorables, comparables quelle que soit la cathode, le remplissage est toujours constitué d'argon $(6660 \mathrm{~Pa})$ et de vapeur de méthylal (1 $332 \mathrm{~Pa})$.

L'électronique associée est très simple; elle comporte l'alimentation de haute tension et une échelle de comptage. L'amplitude de l'impulsion est suffisante pour éviter toute amplification.

\section{SOURCES UTILISÉES - ÉTALONNAGES PRÉALABLES}

Dans le cas des fluences faibles, nous avons employé la source du $\mathrm{Pu}-\alpha-\mathrm{Be}$ ( $7 \mathrm{Ci}$ ) du laboratoire, entourée d'une sphère de paraffine de $18 \mathrm{~cm}$ de diamètre, pour laquelle la thermalisation des neutrons est optimale. La figure 2 précise

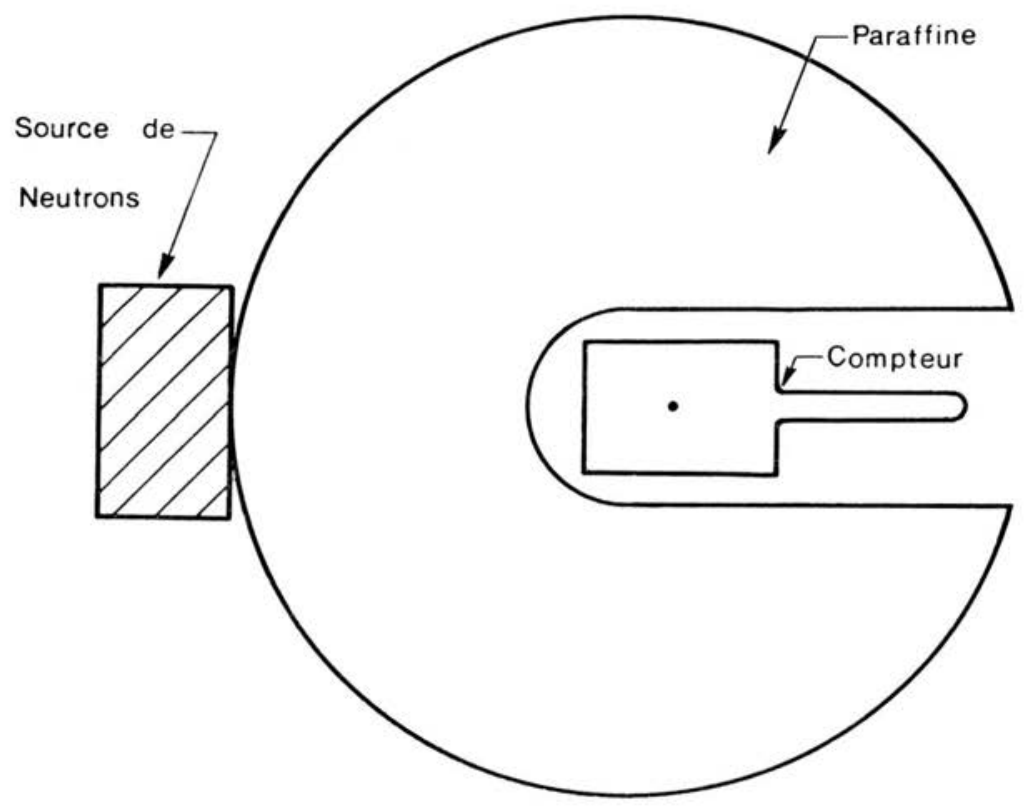

FIg. 2. - Dispositif d'irradiation du détecteur par la source $(\mathrm{Pu}-\alpha-\mathrm{Be})$

les conditions géométriques d'irradiations. Au niveau de la cathode, la valeur moyenne de la fluence est de $4000 \mathrm{n} \mathrm{cm}^{-2} \mathrm{~s}^{-1}$.

Dans le cas de fluences élevées, les irradiations ont eu lieu près du réacteur Siloette du Centre d'Etudes Nucléaires de Grenoble; au niveau de la cathode, les fluences pouvaient être réglées entre $3.10^{4} \mathrm{n} \mathrm{cm}^{-2} \mathrm{~s}^{-1}$ et $2: 75 \cdot 10^{8} \mathrm{n} \mathrm{cm}^{-2} \mathrm{~s}^{-1}$.

Il est indispensable de connaitre les fluences pour obtenir les performances du détecteur. Dans ce but, nous avons utilisé la méthode classique d'activation de feuilles d'or (diamètre : $1 \mathrm{~cm}$, épaisseur : $0,025 \mathrm{~mm}$ ou $0,1 \mathrm{~mm}$ ) irradiées à nu ou sous une épaisseur de cadmium de $1 \mathrm{~mm}$. Le cadmium absorbe les neutrons 
d'énergies inférieures à $0,68 \mathrm{eV}$, dans les conditions de l'opérations [ 12 ]. La contribution des neutrons d'énergies inférieures à $0,68 \mathrm{eV}$ s'obtient par différence de l'activité de la feuille irradiée à nu et sous cadmium. L'activité absolue est obtenue par coïncidences $(\beta-\gamma)[7]$. Un calcul simple donne la fluence [6], après avoir effectué les corrections dues à la perturbation du flux, connaissant les constantes nucléaires de l'or (section efficace, période, etc.) [s].

Enfin, nous avons calculé la dose absorbée en estimant qu'un neutron thermique par $\mathrm{cm}^{2}$ produit une dose de $6.10^{-10} \mathrm{rad}$ dans le tissu biologique mou [ $\mathrm{r}$.

\section{GAMME DE FLUX ET DE DOSES INTÉGRÉS MESURABLES (Tableau 3)}

Nos expériences permettent de souligner un certain nombre de points importants :

I. Le " rapport Cadmium " de nos compteurs (rapport de l'activité obtenue à nu à celle obtenue sous écran de cadmium) est meilleur que celui des feuilles d'or; nous avons obtenu 30 , i 16 et 170 pour les cathodes d'or, de cuivre et de dysprosium, respectivement, contre in, 5 pour une feuille d'or de $\mathrm{I} \mathrm{cm}$ de diamètre, épaisse de $0,025 \mathrm{~mm}$ (l'irradiation se faisant dans des conditions identiques). Autrement dit, dans nos conditions d'irradiation, la contribution des neutrons d'énergies supérieures à $0,68 \mathrm{eV}$ est de $3,33 \%, 0,86 \%$ et $0,59 \%$ pour les cathodes d'or, de cuivre et de dysprosium, contre $8,7 \%$ pour la feuille d'or.

2. Pour éliminer les radionucléides parasites de courtes périodes, il faut attendre, après l'irradiation, un certain temps avant d'effectuer les mesures, dans

TABLEAU 3

RÉSUMÉ DES RÉSULTATS OBTENUS

\begin{tabular}{|c|c|c|c|c|c|}
\hline \multirow[b]{2}{*}{ Cathode } & \multirow[b]{2}{*}{$\begin{array}{c}\text { Flux et dose } \\
\text { minimaux } \\
\text { mesurables } \\
\left(n \mathrm{~cm}^{-2} \text { et rad }\right)\end{array}$} & \multirow[b]{2}{*}{$\begin{array}{c}\text { Début } \\
\text { du comptage } \\
\text { après } \\
\text { irradiation }\end{array}$} & \multirow[b]{2}{*}{$\begin{array}{c}\text { Siloette } \\
\text { Flux et dose } \\
\text { maximaux } \\
\text { mesurables } \\
\left(n \mathrm{~cm}^{-2}, \mathrm{rad}\right) \\
\text { (I) }\end{array}$} & \multicolumn{2}{|c|}{ Source Pu- $\alpha-\mathrm{Be}$} \\
\hline & & & & $\begin{array}{c}\text { Durée } \\
\text { maximale } \\
\text { d'irradiation }\end{array}$ & $\begin{array}{c}\text { Flux et dose } \\
\text { maximaux } \\
\text { mesurables } \\
\left(n \mathrm{~cm}^{-2}, \mathrm{rad}\right) \\
\quad(2)\end{array}$ \\
\hline Dysprosium & $\begin{array}{c}4 \cdot 10^{5} \\
0,25 \cdot 10^{-3}\end{array}$ & Io minutes & $\begin{array}{l}5 \cdot 10^{8} \\
0,3\end{array}$ & 45 minutes & $\begin{array}{l}1,1 \cdot 10^{7} \\
6,5 \cdot 10^{-3}\end{array}$ \\
\hline Or & $\begin{array}{l}5.10^{5} \\
3.10^{-3}\end{array}$ & Immédiat & $\begin{array}{c}\mathrm{I}, 3 \cdot 10^{10} \\
8\end{array}$ & 20 heures & $\begin{array}{c}25 \cdot 10^{7} \\
150.10^{-3}\end{array}$ \\
\hline Cuivre & $\begin{array}{l}2,5 \cdot 10^{7} \\
1,5 \cdot 10^{-3}\end{array}$ & 40 minutes & $\begin{array}{l}17 \cdot 10^{10} \\
100\end{array}$ & 4 heures & $\begin{array}{l}8,3 \cdot 10^{7} \\
50 \cdot 10^{-3}\end{array}$ \\
\hline
\end{tabular}

(I) Limités par le temps mort du détecteur.

(2) Limités par la durée maximale d'irradiation 
le cas de Dy et Cu. Dans le premier cas, il faut attendre ro minutes pour éliminer ${ }^{165} \mathrm{mDy}(\mathrm{I}, 3 \mathrm{mn})$ dans le second 40 minutes pour éliminer, ${ }^{66} \mathrm{Cu}(5, \mathrm{Is} \mathrm{mn})$; voir le tableau I. Par contre, aucune période parasite n'est observée dans le cas de cathodes en or.

3. Les courbes d'étalonnage sont données sur les figures 3,4 et 5 , en fin d'article. Dans le cas du réacteur Siloette, la durée d'irradiation a toujours été très inférieure à la période $\mathrm{T}$ du radionucléide formé : le taux de comptage est proportionnel au flux intégré; la valeur maximale mesurable est limitée par le temps mort $d u$ compteur. Nous prenons comme valeur maximale mesurable celle correspondant à une perte de comptage de $20 \%$ (tableau 3 ).

Dans le cas de la source Pu- $\alpha$-Be, l'irradiation dure plus de $0,3 \mathrm{I}_{3} \mathrm{~T}$, valeur pour laquelle le taux de comptage n'est plus proportionnel au flux intégré, à ro \% près. Dans ce cas, la valeur maximale mesurable est limitée par la durée d'irradiation, qui ne doit pas dépasser 20 heures dans le cas de l'or, 4 heures dans celui du cuivre, 45 minutes dans celui du dysprosium.

Enfin, la valeur minimale mesurable correspond à un taux de comptage, égal à 3 fois le bruit de fond du détecteur (voir le tableau 2).

\section{CONCLUSION.}

Les détecteurs à cathodes planes, robustes, sont adaptables à divers problèmes :

a) Dans le cas de "flashes » de neutrons, ou d'irradiation de courte durée, les cathodes de cuivre conviennent pour les doses élevées, celles de dysprosium pour les doses faibles.

b) Pour des irradiations de longue durée, les cathodes d'or donnent la sensibilité optimale.

Afin de couvrir la gamme complète des flux et des doses du tableau 3, il est possible de placer dans la même enceinte un système comportant trois anodes et trois disques, de cuivre, de dysprosium et d'or.

Ces recherches ont été effectuées sous la direction de M. Daniel Blanc, Directeur du Centre. nous lui exprimons notre reconnaissance. Ce travail a bénéficié d'un contrat de la D.R.M.E,; nous remercions vivement MM. Mays et Malardel pour l'aide qu'ils nous ont apportée, et pour les conseils qu'ils nous ont prodigués. Enfin, nous remercions la Direction du Centre d'Etudes Nucléaires de Grenoble qui nous a permis d'expérimenter près du réacteur Siloette.

\section{BIBLIOGRAPHIE}

[I] Arnaud (Y.). - Neutron dosimetry, Vol. I, AIEA, Vienne (1963), 277.

[2] Bayle (P.), Rakotondrafara (H.), Lévy (M.) et Vallée (A.). - Rev. Pbys. Appl. (1966), I, 97.

[3] Beauge (R.). - Sections efficaces pour les détecteurs de neutrons par activation, Groupe de dosimétrie, EURATOM (1962).

[4] Blanc (D.). - Détecteurs de particules; compteurs et scintillateurs, Masson, Paris (1959).

[s] Brisbors (J.), Lott (M.) et MANent (G.). - Rapport CEA, no R-249I (1964).

[6] Darrès (M.). - Doctorat de spécialité (physique nucléaire), Toulouse, février (1968). 
[7] GANDY (A.). - Préparation et étalonnage des sources radioactives de référence, AIEA, collection "monographies ", no 14, Int. Journ. Applied Rad. and Isotope (1961), 11, 75.

[8] Hanna (G.C.). - Trans. Amer. Nucl. Soc. (ig62), 5, ir.

[9] Rakotondrafara (H.). - Doctorat de spécialité (physique nucléaire), Toulouse, n $\mathrm{n}^{\circ} \mathrm{4}^{\mathrm{I}} \mathrm{s}$ (1966).

[ro] Rirchie (R.H.) et Eldridge (H.B.). - Nucl. Sci. and Eng. (1960), 8, 300.

[i I] SolA (A.). - Nucleonics (1960), $18, \mathrm{n}^{\circ} 3,78$.

[12] Stoughton (R.W.). - Nucl. Sci. Eng. (1963), 15, 314.

[13] Titrle (C.W.). - Nucleonics (1951), 8, no 6, 5 .

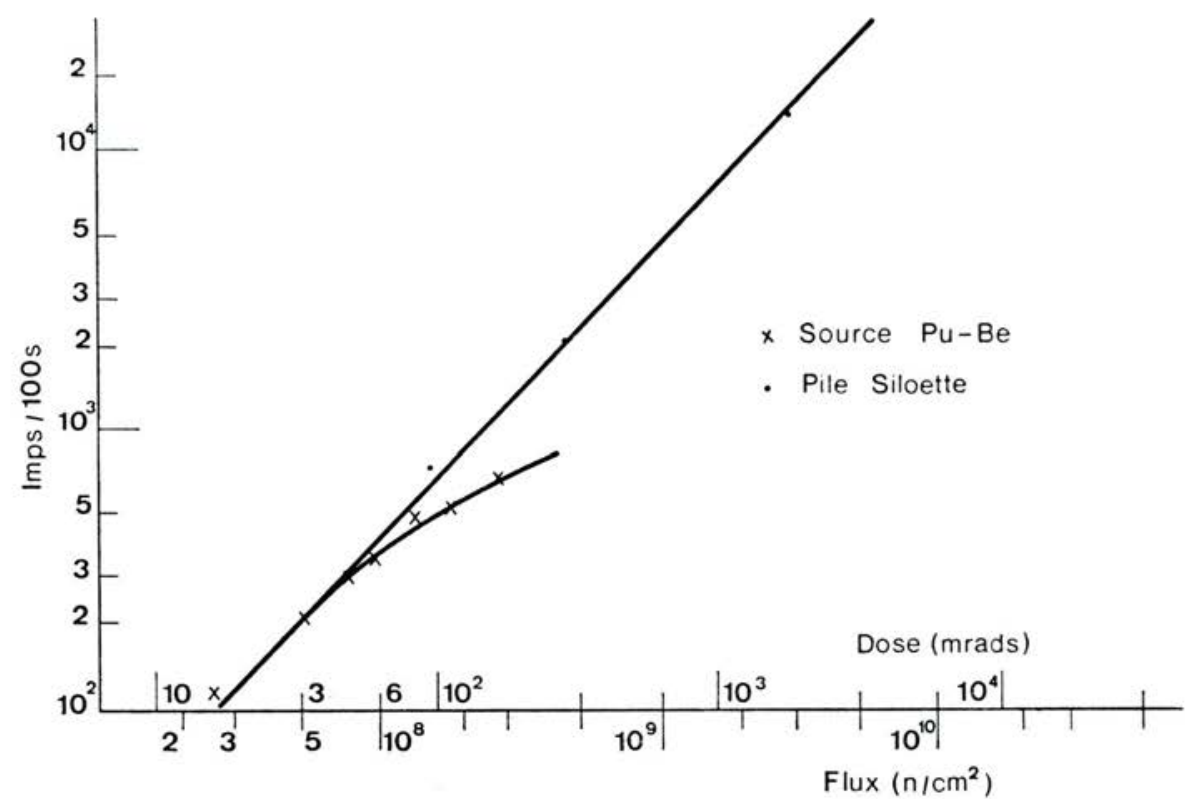

FIG. 3. - Taux de comptage en fonction du flux et de la dose intégrés, pour les cathodes de cuivre (40 minutes après irradiation). 


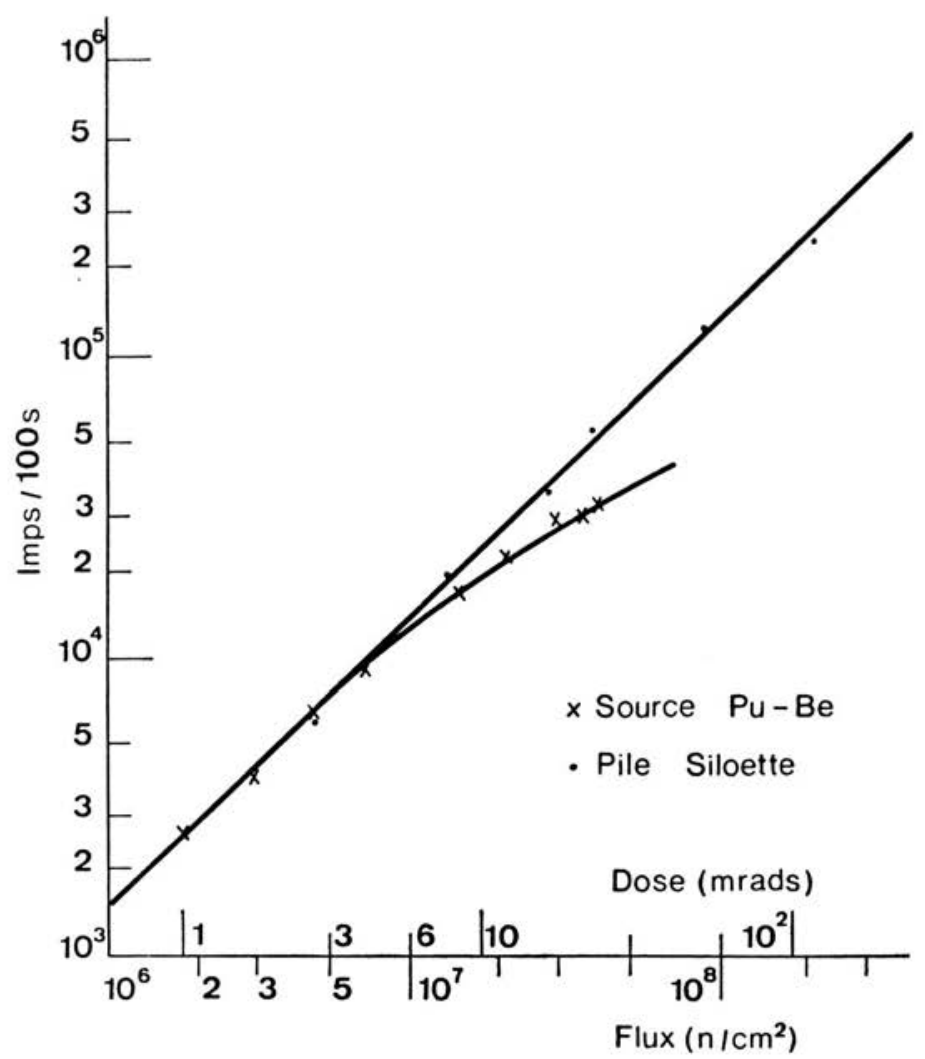

Fig. 4. - Taux de comptage en fonction du flux et de la dose intégrés, pour les cathodes de dysprosium (ro minutes après irradiation). 


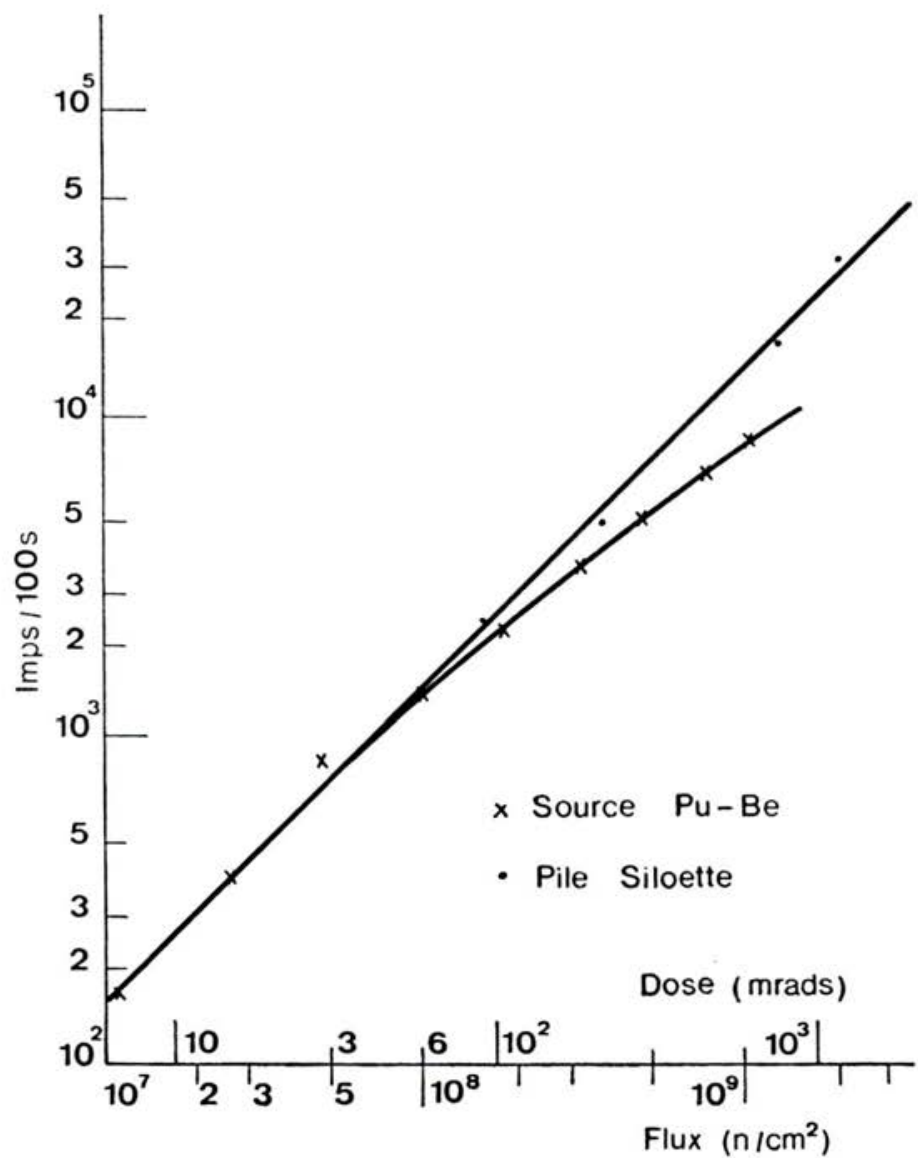

Fig. 5. - Taux de comptage en fonction du flux et de la dose intégrés, pour les cathodes d'or (immédiatement après irradiation). 